\title{
SUPPLEMENTARY MATERIAL \\ Sustainable Design of an Optimal Supply Chain for Furfural Production from Agricultural Wastes
}

\begin{abstract}
Gabriel Contreras-Zarazúa, ${ }^{1}$ Mariano Martin ${ }^{2}$, José María Ponce-Ortega ${ }^{3}$, Juan Gabriel SegoviaHernández ${ }^{1}$

1 Department of Chemical Engineering University of Guanajuato, Noria Alta S/N,36000, Guanajuato,Gto., Mexico.

2 Department of Chemical Engineering, University of Salamanca, Plz. Caídos 1-5, 37008, Salamanca, Spain.

${ }^{3}$ Chemical Engineering Department, Universidad Michoacana de San Nicolás de Hidalgo, Morelia, Michoacán 58060, Mexico
\end{abstract}
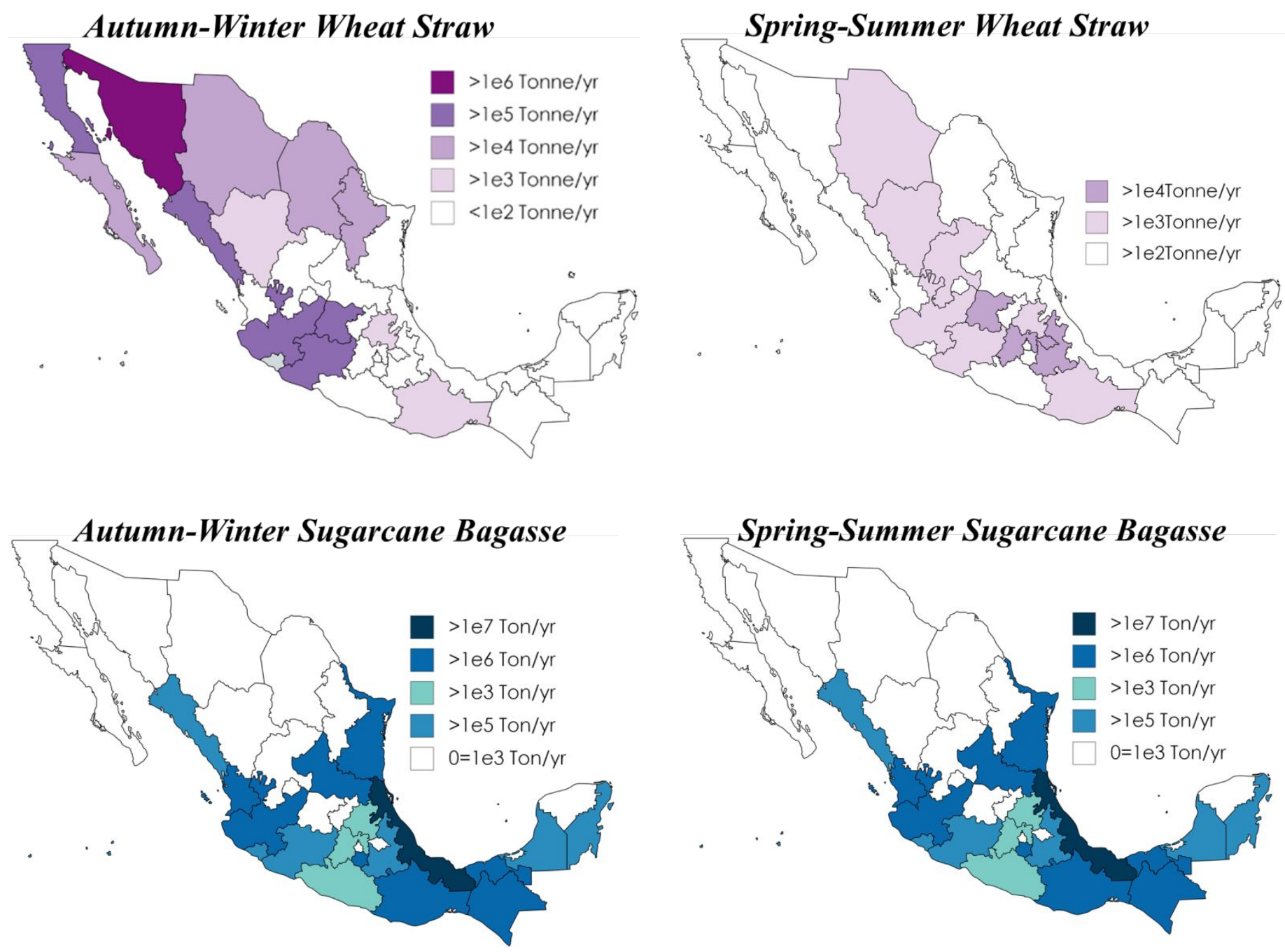

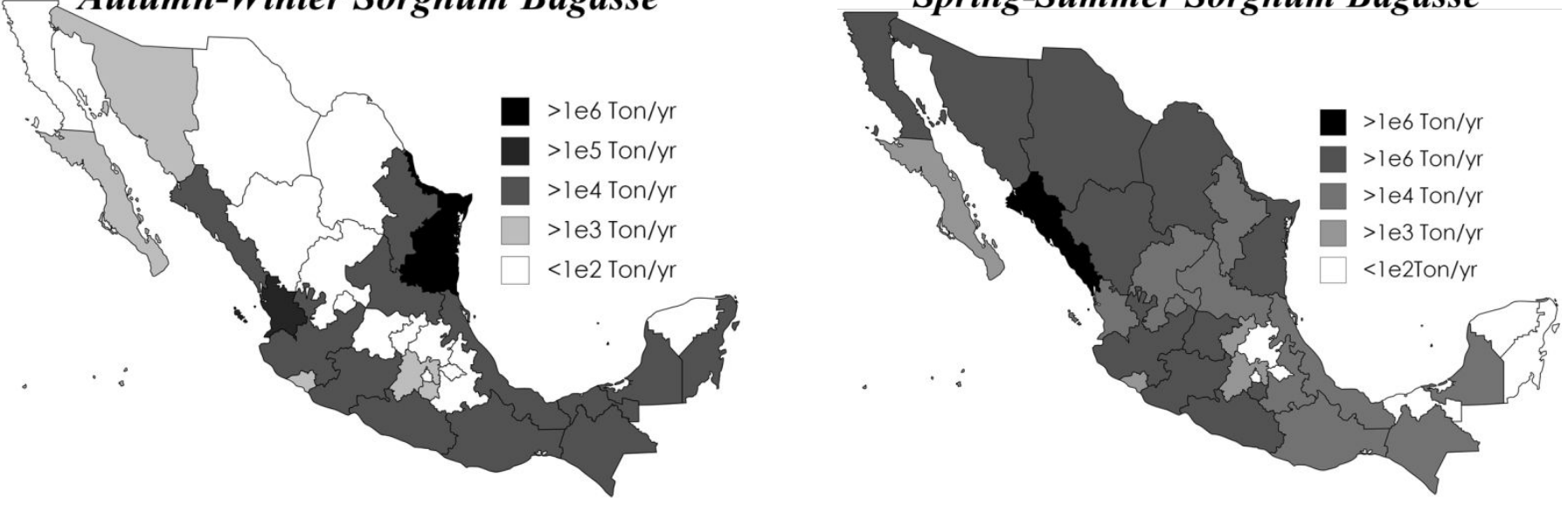

Figure S1. Biomass availability maps for all the raw materials.

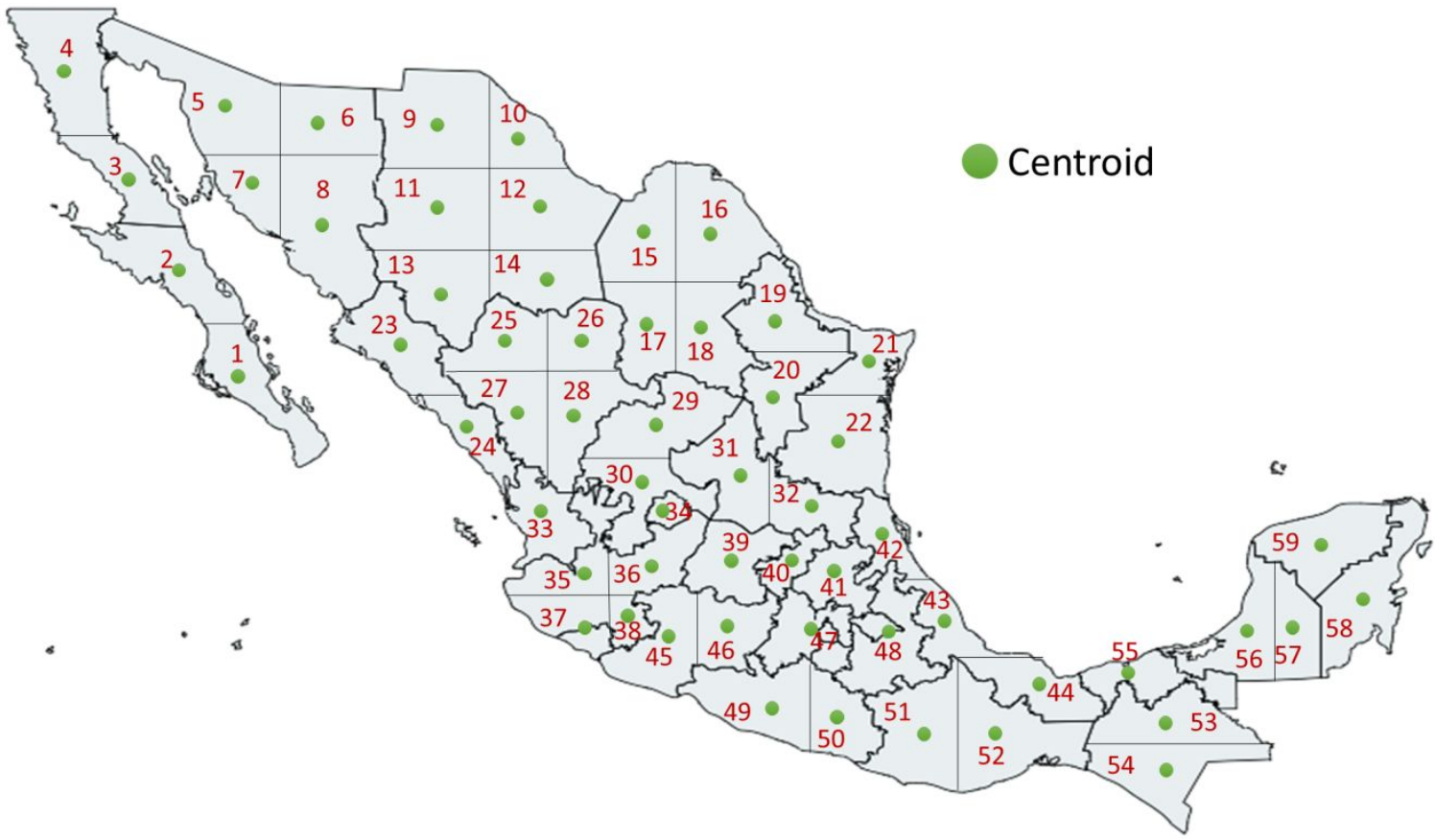

Figure S2. Discretization of Mexico in 59 zones.

\section{Calculation of transportation distances.}

One of the most important points in a supply chain is the calculation of transportation distances. Regarding, the locations of harvest and markets are known as well as, the probable locations biorefineries are know too. These locations are given by the centroid of each zone. The Rhumb line method is used to set the harvest, biorefineries and markets sites locations, in addition, it is used to calculate the transportation distances required ${ }^{1}$. The Rhumb line method is a very common technique used to estimate locations and distances in cartography, it requires only 
latitude $(\sigma)$ and longitude $(\phi)$ data, which can be obtained from Google maps or other programs like ArcGis. This method consists in the transformation of certain area of earth's spheroid into Cartesian projection. This, Cartesian projection requires a reference point called source point or point 0 , which takes the role of coordinate $(0,0)$ in the cartesian projection. The locations of farms, markets and probable refineries are calculated using this point 0 as reference, all these locations are represented with $\mathrm{y}$ and $\mathrm{x}$ coordinates. The determination of $\mathrm{x}$ and $\mathrm{y}$ coordinates for harvest sites, markers and biorefineries are given by the following equations:

$$
\begin{gathered}
x_{1}=\omega\left[\cos \left(\frac{\sigma_{1}+\sigma_{0}}{2}\right)\right]\left(\phi_{1}-\phi_{2}\right) \\
y_{1}=\omega\left(\sigma_{1}+\sigma_{0}\right)
\end{gathered}
$$

Where, $\omega$ represents the radius of earth which corresponds to $6371 \mathrm{~km}, \sigma_{1}$ and $\phi_{1}$ are the centroids latitude and longitude respectively of discretization zones. Finally, $x_{1}$ and $y_{1}$ are the cartesian coordinates of centroids obtained by the rhumb line technique. A graphical representation of rhumb line technique is given by Figure S3. The data of latitude and longitude for harvest, refineries and markets are given in the Tables S5-S7, respectively.

Once, the coordinates of harvest, markets and biorefineries are obtained, the transportation distance can be calculated using a common method as Pythagoras's theorem or other technique. Data from Ministry of Communications and Transports of Mexico (SCT), indicate that each year in Mexico $71 \%$ of total products (200, 000 Mton/year) is transported by road using trucks, $19 \%$ using rail and the rest by airplane and vessel ${ }^{2}$. Based on the previous data, this work considers that the transportation of agricultural wastes and furfural is carry out by road, because it is the most common way to transport products in Mexico. In this way, the method used to calculate the distances between different locations is the rectilinear distance, this method is chosen because it provides a good approximation of transportation distance by roadway, even often the roadways are designed based in rectilinear distances ${ }^{3}$. The rectilinear distances are calculated as follows:

$$
\begin{gathered}
\Delta x_{1,2}=\left|x_{1}-x_{2}\right| \\
\Delta y_{1,2}=\left|y_{1}-y_{2}\right| \\
D_{1,2}=\Delta x_{1,2}+\Delta y_{1,2}
\end{gathered}
$$




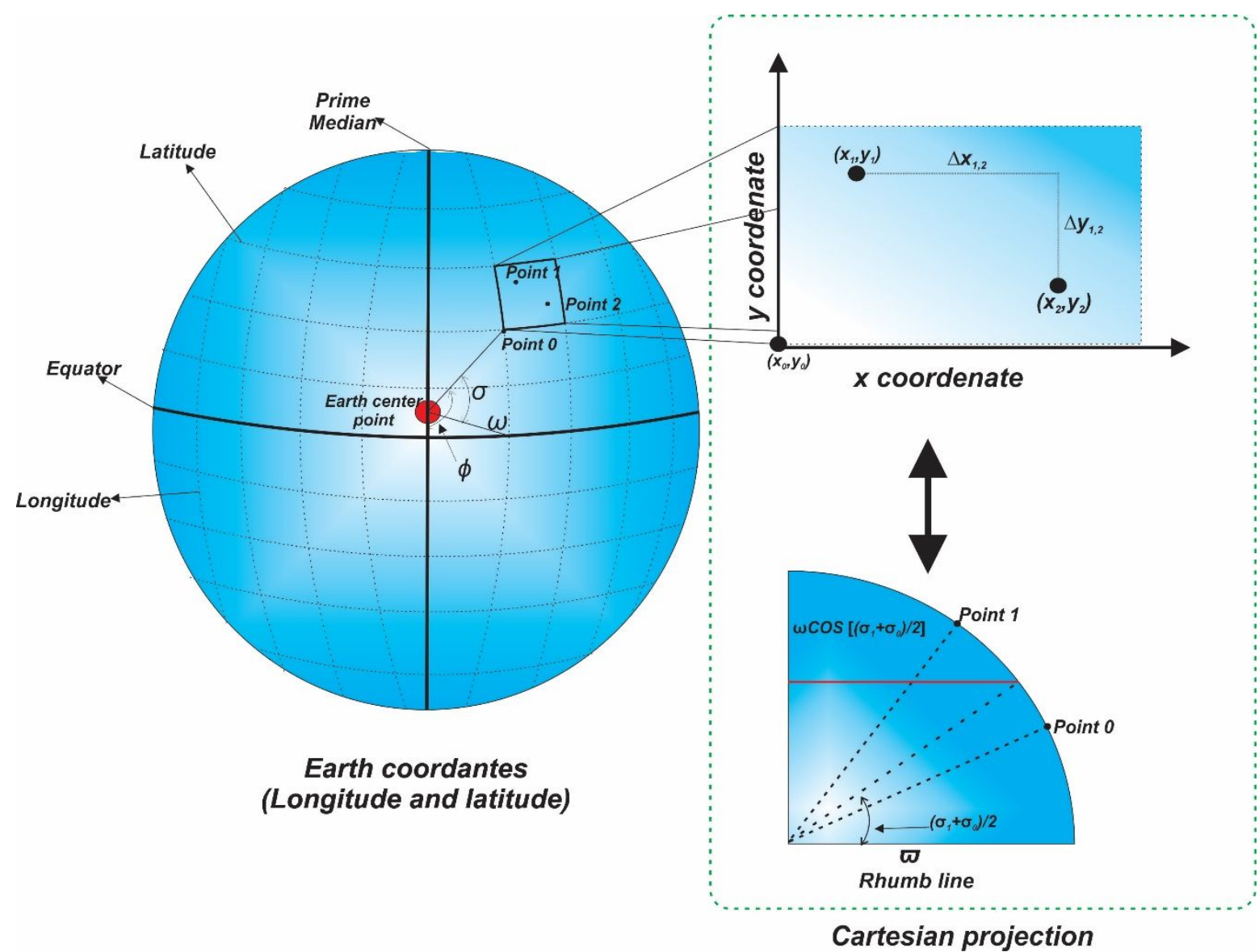

Figure S3. Transformation of locations coordinates to a cartesian plane. 


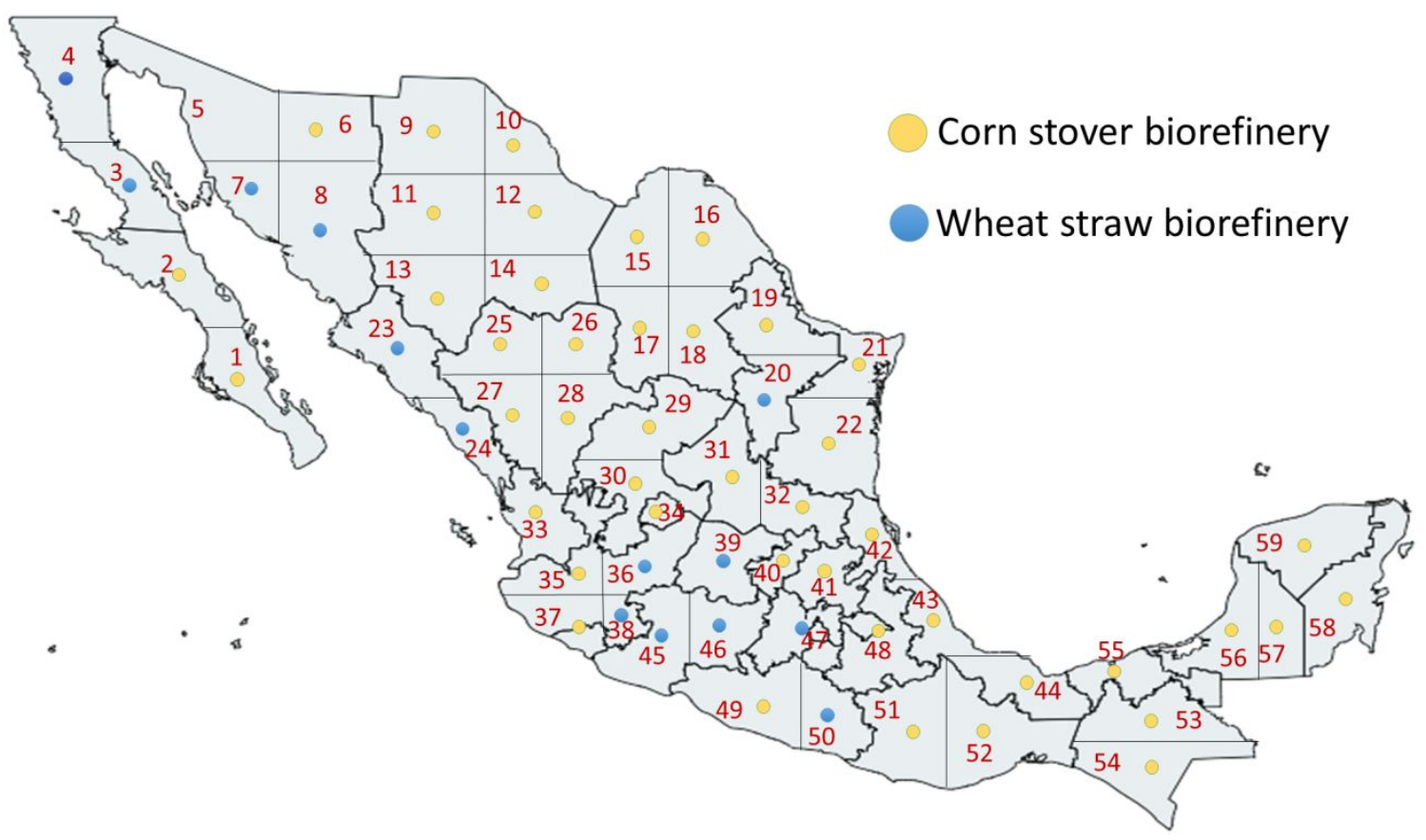

Figure S4. Furfural Biorefineries locations.

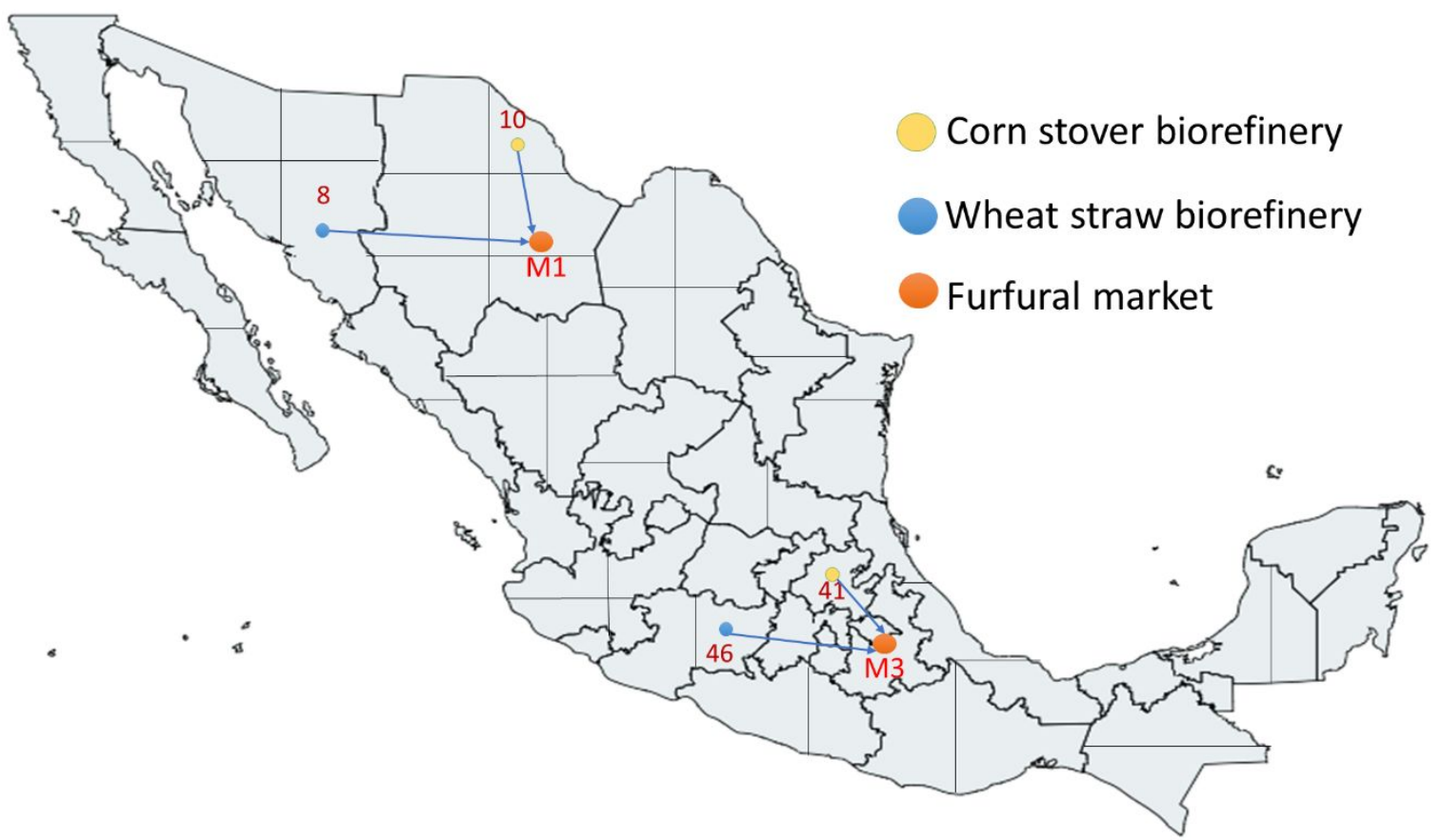

Figure S5. Optimal supply chain of furfural for markets 1 and 3. 


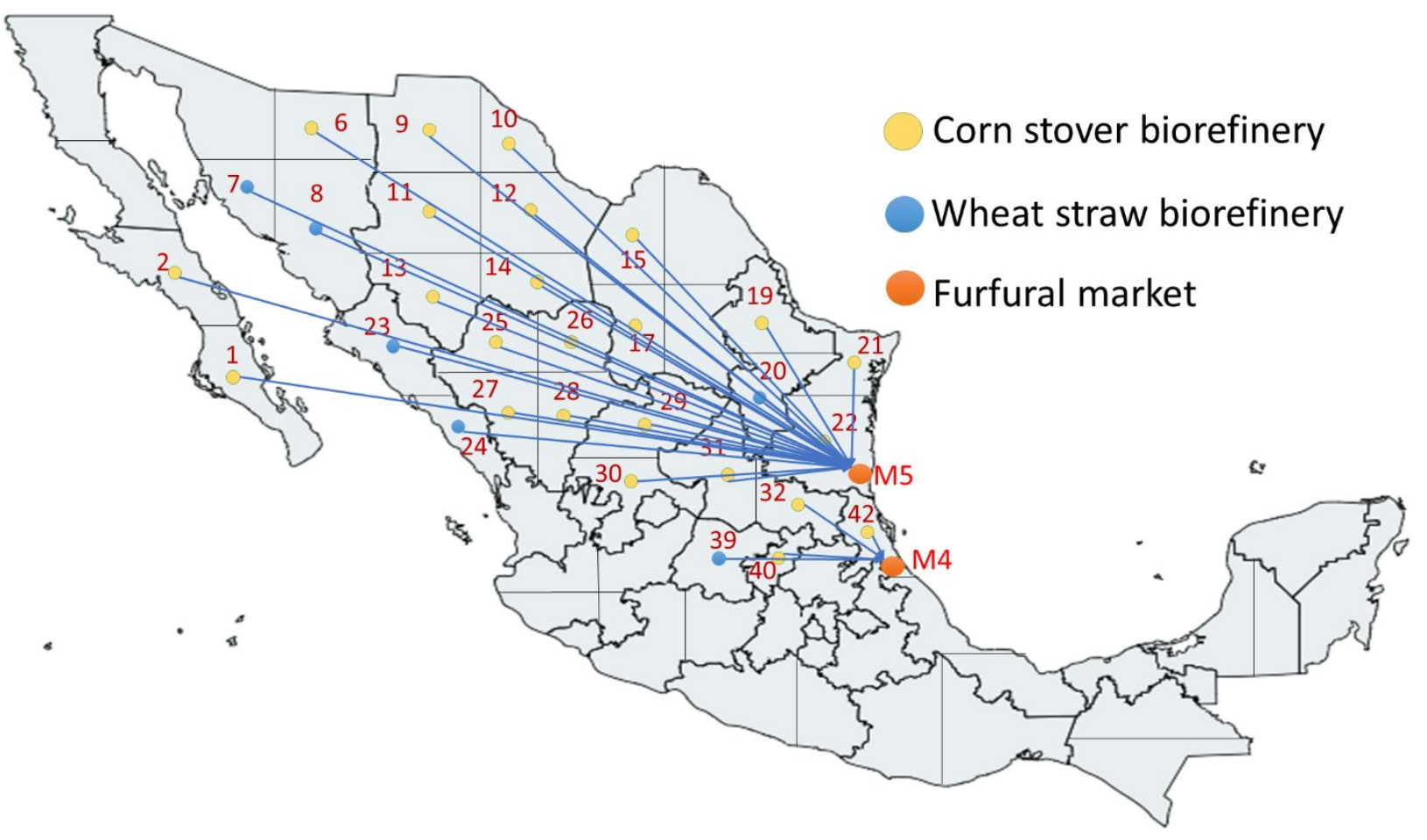

Figure S6. Optimal supply chain of furfural for markets 4 and 5 .

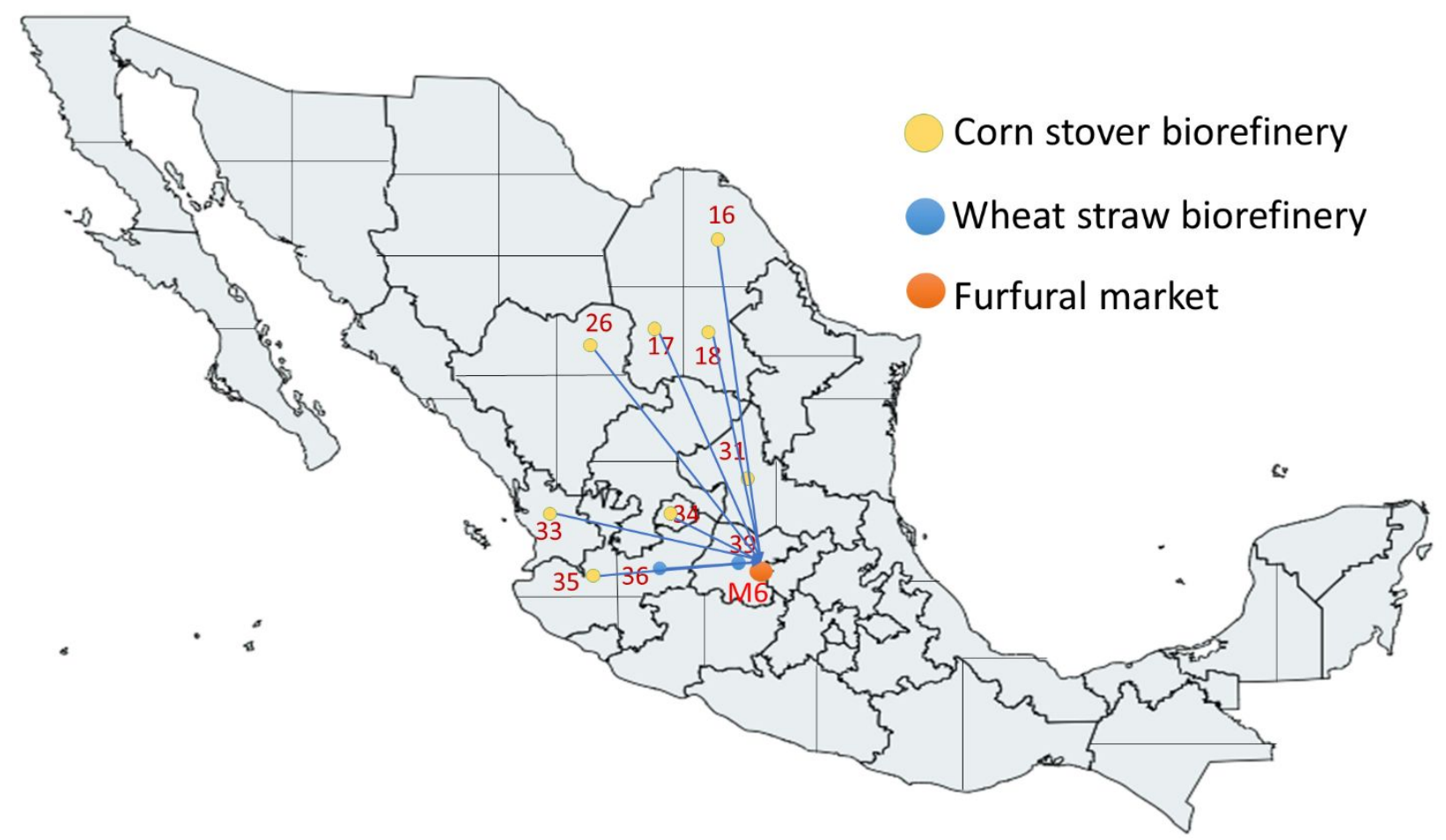

Figure S7. Optimal supply chain of furfural for market 6 . 


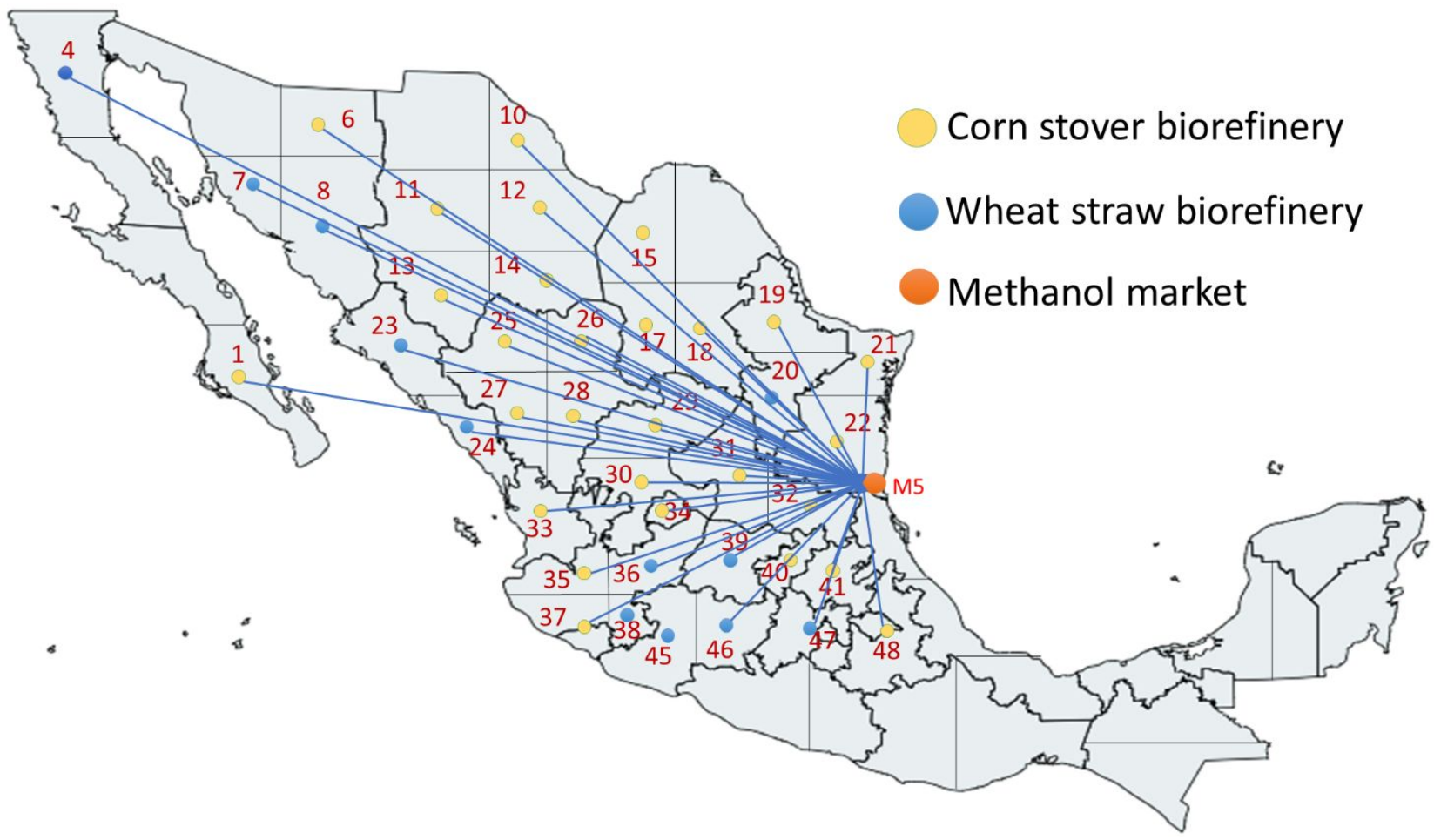

Figure S9. Optimal supply chain of methanol for market 5. 
Table S1. Biomass availability in metric tons during spring season.

\begin{tabular}{|c|c|c|c|c|}
\hline Zone & Corn Stover & $\begin{array}{l}\text { Wheat } \\
\text { Straw }\end{array}$ & $\begin{array}{c}\text { Sorghum } \\
\text { Bagasse }\end{array}$ & $\begin{array}{c}\text { Sugarcane } \\
\text { Bagasse }\end{array}$ \\
\hline $\mathrm{H} 1$ & 627 & 0 & 0 & 0 \\
\hline $\mathrm{H} 2$ & 6076 & 0 & 2087 & 0 \\
\hline $\mathrm{H} 3$ & 0 & 0 & 21252 & 0 \\
\hline $\mathrm{H} 4$ & 9981 & 0 & 49589 & 0 \\
\hline H5 & 20 & 0 & 20731 & 0 \\
\hline H6 & 2729 & 0 & 59231 & 0 \\
\hline $\mathrm{H} 7$ & 2402 & 0 & 0 & 0 \\
\hline $\mathrm{H} 8$ & 10841 & 0 & 117475 & 238 \\
\hline H9 & 350230 & 0 & 7025 & 0 \\
\hline $\mathrm{H} 10$ & 81453 & 0 & 2810 & 0 \\
\hline H11 & 402839 & 306 & 4215 & 0 \\
\hline $\mathrm{H} 12$ & 286167 & 306 & 28098 & 0 \\
\hline $\mathrm{H} 13$ & 9474 & 715 & 42147 & 0 \\
\hline H14 & 90132 & 715 & 56196 & 0 \\
\hline H15 & 33771 & 0 & 37258 & 0 \\
\hline H16 & 5955 & 0 & 55886 & 0 \\
\hline $\mathrm{H} 17$ & 530939 & 0 & 111773 & 0 \\
\hline $\mathrm{H} 18$ & 9011 & 174 & 167659 & 0 \\
\hline H19 & 937 & 0 & 6098 & 0 \\
\hline $\mathrm{H} 20$ & 23783 & 51 & 16488 & 0 \\
\hline $\mathrm{H} 21$ & 3256 & 0 & 15350 & 215631 \\
\hline $\mathrm{H} 22$ & 12912 & 0 & 40469 & 503139 \\
\hline $\mathrm{H} 23$ & 359023 & 0 & 260036 & 7040 \\
\hline $\mathrm{H} 24$ & 5608 & 0 & 390054 & 63356 \\
\hline $\mathrm{H} 25$ & 56858 & 655 & 54739 & 0 \\
\hline $\mathrm{H} 26$ & 867990 & 655 & 46953 & 0 \\
\hline $\mathrm{H} 27$ & 123919 & 1223 & 64956 & 0 \\
\hline $\mathrm{H} 28$ & 483730 & 1834 & 76634 & 0 \\
\hline $\mathrm{H} 29$ & 281344 & 995 & 6497 & 0 \\
\hline $\mathrm{H} 30$ & 1002530 & 3981 & 10204 & 379 \\
\hline $\mathrm{H} 31$ & 68831 & 0 & 7319 & 438094 \\
\hline $\mathrm{H} 32$ & 19473 & 26 & 4879 & 657141 \\
\hline H33 & 176180 & 0 & 11525 & 542149 \\
\hline H34 & 814499 & 0 & 13051 & 0 \\
\hline H35 & 1204754 & 0 & 39111 & 632277 \\
\hline $\mathrm{H} 36$ & 3279882 & 766 & 58667 & 270976 \\
\hline $\mathrm{H} 37$ & 209005 & 0 & 32767 & 891800 \\
\hline H38 & 1136767 & 511 & 68445 & 270976 \\
\hline H39 & 1139712 & 11161 & 428901 & 25 \\
\hline $\mathrm{H} 40$ & 544846 & 73 & 4687 & 0 \\
\hline $\mathrm{H} 41$ & 444800 & 660 & 161 & 9694 \\
\hline $\mathrm{H} 42$ & 74774 & 0 & 7067 & 1064141 \\
\hline $\mathrm{H} 43$ & 138920 & 383 & 3029 & 1596212 \\
\hline
\end{tabular}




\begin{tabular}{c|cccc}
\hline H44 & 185967 & 0 & 0 & 2660353 \\
H45 & 346613 & 222 & 98989 & 321241 \\
H46 & 710854 & 519 & 142448 & 35693 \\
H47 & 1632928 & 33616 & 90418 & 564753 \\
H48 & 1031557 & 8298 & 40677 & 464487 \\
H49 & 386868 & 0 & 34580 & 1657 \\
H50 & 242856 & 0 & 51871 & 1657 \\
H51 & 183711 & 4313 & 26712 & 381742 \\
H52 & 115324 & 0 & 0 & 572612 \\
H53 & 364953 & 24 & 7627 & 300787 \\
H54 & 156409 & 0 & 0 & 451180 \\
H55 & 46152 & 0 & 477 & 640578 \\
H56 & 238451 & 0 & 6188 & 216036 \\
H57 & 0 & 0 & 0 & 0 \\
H58 & 53509 & 0 & 139 & 151 \\
H59 & 36357 & 0 & 58 & 434186 \\
\hline
\end{tabular}

Table S2. Biomass availability in metric tons during summer season.

\begin{tabular}{c|cccc}
\hline Zone & Corn Stover & $\begin{array}{c}\text { Wheat } \\
\text { Straw }\end{array}$ & $\begin{array}{c}\text { Sorghum } \\
\text { Bagasse }\end{array}$ & $\begin{array}{c}\text { Sugarcane } \\
\text { Bagasse }\end{array}$ \\
\hline H1 & 627 & 0 & 0 & 0 \\
H2 & 6076 & 0 & 2087 & 0 \\
H3 & 0 & 0 & 21252 & 0 \\
H4 & 9981 & 0 & 49589 & 0 \\
H5 & 20 & 0 & 20731 & 0 \\
H6 & 2729 & 0 & 59231 & 0 \\
H7 & 2402 & 0 & 0 & 0 \\
H8 & 10841 & 0 & 117475 & 238 \\
H9 & 350230 & 0 & 7025 & 0 \\
H10 & 81453 & 0 & 2810 & 0 \\
H11 & 402839 & 306 & 4215 & 0 \\
H12 & 286167 & 306 & 28098 & 0 \\
H13 & 9474 & 715 & 42147 & 0 \\
H14 & 90132 & 715 & 56196 & 0 \\
H15 & 33771 & 0 & 37258 & 0 \\
H16 & 5955 & 0 & 55886 & 0 \\
H17 & 530939 & 0 & 111773 & 0 \\
H18 & 9011 & 174 & 167659 & 0 \\
H19 & 937 & 0 & 6098 & 0 \\
H20 & 23783 & 51 & 16488 & 0 \\
H21 & 3256 & 0 & 15350 & 215631 \\
H22 & 12912 & 0 & 40469 & 503139 \\
H23 & 359023 & 0 & 260036 & 7040 \\
H24 & 5608 & 0 & 390054 & 63356 \\
H25 & 56858 & 655 & 54739 & 0 \\
\hline & & & & \\
\hline
\end{tabular}




\begin{tabular}{|c|c|c|c|c|}
\hline $\mathrm{H} 26$ & 867990 & 655 & 46953 & 0 \\
\hline $\mathrm{H} 27$ & 123919 & 1223 & 64956 & 0 \\
\hline $\mathrm{H} 28$ & 483730 & 1834 & 76634 & 0 \\
\hline $\mathrm{H} 29$ & 281344 & 995 & 6497 & 0 \\
\hline $\mathrm{H} 30$ & 1002530 & 3981 & 10204 & 379 \\
\hline H31 & 68831 & 0 & 7319 & 438094 \\
\hline H32 & 19473 & 26 & 4879 & 657141 \\
\hline H33 & 176180 & 0 & 11525 & 542149 \\
\hline H34 & 814499 & 0 & 13051 & 0 \\
\hline H35 & 1204754 & 0 & 39111 & 632277 \\
\hline H36 & 3279882 & 766 & 58667 & 270976 \\
\hline H37 & 209005 & 0 & 32767 & 891800 \\
\hline H38 & 1136767 & 511 & 68445 & 270976 \\
\hline H39 & 1139712 & 11161 & 428901 & 25 \\
\hline $\mathrm{H} 40$ & 544846 & 73 & 4687 & 0 \\
\hline H41 & 444800 & 660 & 161 & 9694 \\
\hline $\mathrm{H} 42$ & 74774 & 0 & 7067 & 1064141 \\
\hline $\mathrm{H} 43$ & 138920 & 383 & 3029 & 1596212 \\
\hline H44 & 185967 & 0 & 0 & 2660353 \\
\hline $\mathrm{H} 45$ & 346613 & 222 & 98989 & 321241 \\
\hline H46 & 710854 & 519 & 142448 & 35693 \\
\hline $\mathrm{H} 47$ & 1632928 & 33616 & 90418 & 564753 \\
\hline $\mathrm{H} 48$ & 1031557 & 8298 & 40677 & 464487 \\
\hline H49 & 386868 & 0 & 34580 & 1657 \\
\hline H50 & 242856 & 0 & 51871 & 1657 \\
\hline H51 & 183711 & 4313 & 26712 & 381742 \\
\hline H52 & 115324 & 0 & 0 & 572612 \\
\hline H53 & 364953 & 24 & 7627 & 300787 \\
\hline H54 & 156409 & 0 & 0 & 451180 \\
\hline H55 & 46152 & 0 & 477 & 640578 \\
\hline H56 & 238451 & 0 & 6188 & 216036 \\
\hline H57 & 0 & 0 & 0 & 0 \\
\hline H58 & 53509 & 0 & 139 & 151 \\
\hline H59 & 36357 & 0 & 58 & 434186 \\
\hline
\end{tabular}


Table S3. Biomass availability in metric tons during autumn season.

\begin{tabular}{|c|c|c|c|c|}
\hline Zone & Corn Stover & $\begin{array}{l}\text { Wheat } \\
\text { Straw }\end{array}$ & $\begin{array}{c}\text { Sorghum } \\
\text { Bagasse }\end{array}$ & $\begin{array}{c}\text { Sugarcane } \\
\text { Bagasse } \\
\end{array}$ \\
\hline $\mathrm{H} 1$ & 1709 & 0 & 0 & 0 \\
\hline $\mathrm{H} 2$ & 17278 & 6516 & 2939 & 0 \\
\hline H3 & 0 & 0 & 0 & 0 \\
\hline $\mathrm{H} 4$ & 1252 & 137194 & 51 & 0 \\
\hline H5 & 0 & 0 & 458 & 0 \\
\hline H6 & 0 & 0 & 1309 & 0 \\
\hline $\mathrm{H} 7$ & 0 & 211255 & 0 & 0 \\
\hline H8 & 0 & 492928 & 2595 & 238 \\
\hline H9 & 0 & 0 & 0 & 0 \\
\hline H10 & 0 & 0 & 0 & 0 \\
\hline H11 & 0 & 3544 & 0 & 0 \\
\hline H12 & 0 & 3544 & 0 & 0 \\
\hline H13 & 0 & 8270 & 0 & 0 \\
\hline H14 & 0 & 8270 & 0 & 0 \\
\hline H15 & 0 & 0 & 0 & 0 \\
\hline H16 & 0 & 0 & 0 & 0 \\
\hline H17 & 0 & 4437 & 0 & 0 \\
\hline H18 & 0 & 6655 & 0 & 0 \\
\hline H19 & 1352 & 0 & 4384 & 0 \\
\hline $\mathrm{H} 20$ & 329 & 19745 & 11852 & 0 \\
\hline $\mathrm{H} 21$ & 440201 & 0 & 255246 & 215631 \\
\hline $\mathrm{H} 22$ & 60214 & 188 & 672922 & 503139 \\
\hline $\mathrm{H} 23$ & 2435440 & 46812 & 13166 & 7040 \\
\hline $\mathrm{H} 24$ & 341680 & 70218 & 19749 & 63356 \\
\hline $\mathrm{H} 25$ & 41 & 502 & 0 & 0 \\
\hline $\mathrm{H} 26$ & 0 & 502 & 0 & 0 \\
\hline $\mathrm{H} 27$ & 0 & 937 & 0 & 0 \\
\hline $\mathrm{H} 28$ & 0 & 1406 & 0 & 0 \\
\hline $\mathrm{H} 29$ & 0 & 0 & 0 & 0 \\
\hline H30 & 336 & 506 & 58 & 379 \\
\hline H31 & 1434 & 0 & 23990 & 438094 \\
\hline H32 & 43877 & 0 & 15993 & 657141 \\
\hline H33 & 36405 & 0 & 94686 & 542149 \\
\hline H34 & 0 & 0 & 0 & 0 \\
\hline H35 & 3316 & 0 & 3357 & 632277 \\
\hline H36 & 0 & 39652 & 5035 & 270976 \\
\hline $\mathrm{H} 37$ & 14767 & 0 & 5855 & 891800 \\
\hline $\mathrm{H} 38$ & 1695 & 26435 & 5874 & 270976 \\
\hline H39 & 1388 & 168280 & 0 & 25 \\
\hline $\mathrm{H} 40$ & 109 & 0 & 0 & 0 \\
\hline H41 & 22078 & 1433 & 62 & 9694 \\
\hline $\mathrm{H} 42$ & 63513 & 0 & 20958 & 1064141 \\
\hline $\mathrm{H} 43$ & 17088 & 75 & 8982 & 1596212 \\
\hline
\end{tabular}




\begin{tabular}{c|cccc}
\hline H44 & 186402 & 0 & 0 & 2660353 \\
H45 & 14026 & 33071 & 17548 & 321241 \\
H46 & 5128 & 77166 & 25252 & 35693 \\
H47 & 2593 & 268 & 1359 & 564753 \\
H48 & 28884 & 33 & 0 & 464487 \\
H49 & 50527 & 0 & 16511 & 1657 \\
H50 & 10202 & 0 & 24766 & 1657 \\
H51 & 76275 & 1231 & 10260 & 381742 \\
H52 & 15400 & 0 & 0 & 572612 \\
H53 & 68953 & 4 & 7358 & 300787 \\
H54 & 29551 & 0 & 0 & 451180 \\
H55 & 34859 & 0 & 9755 & 640578 \\
H56 & 16369 & 0 & 35628 & 216036 \\
H57 & 0 & 0 & 0 & 0 \\
H58 & 14223 & 0 & 498 & 151 \\
H59 & 3924 & 0 & 6418 & 434186 \\
\hline
\end{tabular}

Table S4. Biomass availability in metric tons during winter season.

\begin{tabular}{c|cccc}
\hline Zone & Corn Stover & $\begin{array}{c}\text { Wheat } \\
\text { Straw }\end{array}$ & $\begin{array}{c}\text { Sorghum } \\
\text { Bagasse }\end{array}$ & $\begin{array}{c}\text { Sugarcane } \\
\text { Bagasse }\end{array}$ \\
\hline H1 & 1709 & 0 & 0 & 0 \\
H2 & 17278 & 6516 & 2939 & 0 \\
H3 & 0 & 0 & 0 & 0 \\
H4 & 1252 & 137194 & 51 & 0 \\
H5 & 0 & 0 & 458 & 0 \\
H6 & 0 & 0 & 1309 & 0 \\
H7 & 0 & 211255 & 0 & 0 \\
H8 & 0 & 492928 & 2595 & 238 \\
H9 & 0 & 0 & 0 & 0 \\
H10 & 0 & 0 & 0 & 0 \\
H11 & 0 & 3544 & 0 & 0 \\
H12 & 0 & 3544 & 0 & 0 \\
H13 & 0 & 8270 & 0 & 0 \\
H14 & 0 & 8270 & 0 & 0 \\
H15 & 0 & 0 & 0 & 0 \\
H16 & 0 & 0 & 0 & 0 \\
H17 & 0 & 4437 & 0 & 0 \\
H18 & 0 & 6655 & 0 & 0 \\
H19 & 1352 & 0 & 4384 & 0 \\
H20 & 329 & 19745 & 11852 & 03139 \\
H21 & 440201 & 0 & 255246 & 040 \\
H22 & 60214 & 188 & 672922 & 0 \\
H23 & 2435440 & 46812 & 13166 & 0 \\
H24 & 341680 & 70218 & 19749 & 0 \\
H25 & 41 & 502 & 0 & 0 \\
\hline & & & & 0 \\
\hline
\end{tabular}




\begin{tabular}{|c|c|c|c|c|}
\hline $\mathrm{H} 26$ & 0 & 502 & 0 & 0 \\
\hline $\mathrm{H} 27$ & 0 & 937 & 0 & 0 \\
\hline $\mathrm{H} 28$ & 0 & 1406 & 0 & 0 \\
\hline H29 & 0 & 0 & 0 & 0 \\
\hline H30 & 336 & 506 & 58 & 379 \\
\hline H31 & 1434 & 0 & 23990 & 438094 \\
\hline H32 & 43877 & 0 & 15993 & 657141 \\
\hline H33 & 36405 & 0 & 94686 & 542149 \\
\hline H34 & 0 & 0 & 0 & 0 \\
\hline H35 & 3316 & 0 & 3357 & 632277 \\
\hline H36 & 0 & 39652 & 5035 & 270976 \\
\hline H37 & 14767 & 0 & 5855 & 891800 \\
\hline H38 & 1695 & 26435 & 5874 & 270976 \\
\hline H39 & 1388 & 168280 & 0 & 25 \\
\hline $\mathrm{H} 40$ & 109 & 0 & 0 & 0 \\
\hline H41 & 22078 & 1433 & 62 & 9694 \\
\hline $\mathrm{H} 42$ & 63513 & 0 & 20958 & 1064141 \\
\hline $\mathrm{H} 43$ & 17088 & 75 & 8982 & 1596212 \\
\hline H44 & 186402 & 0 & 0 & 2660353 \\
\hline H45 & 14026 & 33071 & 17548 & 321241 \\
\hline H46 & 5128 & 77166 & 25252 & 35693 \\
\hline H47 & 2593 & 268 & 1359 & 564753 \\
\hline H48 & 28884 & 33 & 0 & 464487 \\
\hline H49 & 50527 & 0 & 16511 & 1657 \\
\hline $\mathrm{H} 50$ & 10202 & 0 & 24766 & 1657 \\
\hline H51 & 76275 & 1231 & 10260 & 381742 \\
\hline H52 & 15400 & 0 & 0 & 572612 \\
\hline H53 & 68953 & 4 & 7358 & 300787 \\
\hline H54 & 29551 & 0 & 0 & 451180 \\
\hline H55 & 34859 & 0 & 9755 & 640578 \\
\hline H56 & 16369 & 0 & 35628 & 216036 \\
\hline H57 & 0 & 0 & 0 & 0 \\
\hline H58 & 14223 & 0 & 498 & 151 \\
\hline H59 & 3924 & 0 & 6418 & 434186 \\
\hline
\end{tabular}


Table S5. Latitude and longitude of Harvest Sites.

\begin{tabular}{|c|c|c|c|}
\hline State & Zone (Node) & Latitude & Longitude \\
\hline Point $(0,0)$ & Origin point & 10.483034 & -110.481251 \\
\hline \multicolumn{4}{|c|}{ Harvest sites } \\
\hline Baja California sur & H1 & 24.658376 & -111.240604 \\
\hline Baja California sur & $\mathrm{H} 2$ & 27.227629 & -112.858757 \\
\hline Baja California Norte & H3 & 29.306031 & -114.268539 \\
\hline Baja California Norte & $\mathrm{H} 4$ & 31.312156 & -115.634418 \\
\hline Sonora & H5 & 30.7204 & -112.134096 \\
\hline Sonora & H6 & 30.501472 & -109.645912 \\
\hline Sonora & $\mathrm{H} 7$ & 29.121628 & -111.4476 \\
\hline Sonora & H8 & 28.714619 & -109.709545 \\
\hline Chichuahua & H9 & 30.362527 & -107.461827 \\
\hline Chichuahua & H10 & 30.569808 & -105.81517 \\
\hline Chichuahua & H11 & 28.506626 & -107.291241 \\
\hline Chichuahua & H12 & 28.540526 & -104.820946 \\
\hline Chichuahua & H13 & 26.830459 & -107.05085 \\
\hline Chichuahua & H14 & 27.140059 & -104.931007 \\
\hline Coahulia & H15 & 28.338855 & -102.692617 \\
\hline Coahulia & H16 & 27.916192 & -101.169497 \\
\hline Coahulia & H17 & 25.848391 & -102.86979 \\
\hline Coahulia & H18 & 25.864805 & -101.632436 \\
\hline Nuevo León & H19 & 26.478475 & -100.112484 \\
\hline Nuevo León & $\mathrm{H} 20$ & 24.724821 & -100.050375 \\
\hline Tamaulipas & $\mathrm{H} 21$ & 25.069376 & -98.077052 \\
\hline Tamaulipas & $\mathrm{H} 22$ & 23.645813 & -99.10828 \\
\hline Sinaloa & $\mathrm{H} 23$ & 25.459431 & -108.077254 \\
\hline Sinaloa & $\mathrm{H} 24$ & 23.407332 & -106.21131 \\
\hline Durango & $\mathrm{H} 25$ & 25.774991 & -105.781801 \\
\hline Durango & $\mathrm{H} 26$ & 25.862144 & -104.267838 \\
\hline Durango & $\mathrm{H} 27$ & 23.778677 & -105.355204 \\
\hline Durango & $\mathrm{H} 28$ & 24.463302 & -104.189414 \\
\hline Zacatecas & $\mathrm{H} 29$ & 23.832304 & -103.044596 \\
\hline Zacatecas & H30 & 22.648265 & -102.987382 \\
\hline San Luis Potosi & H31 & 22.753378 & -101.072823 \\
\hline San Luis Potosi & H32 & 22.133386 & -99.598359 \\
\hline Nayarit & H33 & 21.501387 & -104.894399 \\
\hline Aguascalientes & H34 & 21.883049 & -102.26628 \\
\hline Guadalajara & H35 & 20.837832 & -103.73164 \\
\hline Guadalajara & H36 & 21.036091 & -102.407509 \\
\hline Guadalajara & H37 & 19.765306 & -104.385153 \\
\hline Guadalajara & H38 & 19.92348 & -103.012252 \\
\hline Guanajuato & H39 & 21.017805 & -101.255714 \\
\hline Queretaro & $\mathrm{H} 40$ & 20.741175 & -99.945244 \\
\hline Hidalgo & H41 & 20.312063 & -98.917813 \\
\hline
\end{tabular}




\begin{tabular}{c|ccc}
\hline Veracruz & H42 & 21.072737 & -97.730163 \\
Veracruz & H43 & 19.204779 & -96.182502 \\
Veracruz & H44 & 17.978818 & -94.91556 \\
Michoacan & H45 & 19.144354 & -102.367499 \\
Michoacan & H46 & 19.796906 & -101.104071 \\
Edo y ciudad de Mexico & H47 & 19.500922 & -99.181979 \\
Puebla y Tlaxcala & H48 & 19.316073 & -97.651463 \\
Guerrero & H49 & 18.35873 & -100.668691 \\
Guerrero & H50 & 17.423789 & -99.486073 \\
Oaxaca & H51 & 17.29984 & -96.709945 \\
Oaxaca & H52 & 16.435997 & -95.023007 \\
Chiapas & H53 & 16.640923 & -93.109964 \\
Chiapas & H54 & 16.294523 & -92.104628 \\
Tabasco & H55 & 18.065858 & -92.874599 \\
Campeche & H56 & 18.67046 & -90.743081 \\
Campeche & H57 & 18.907372 & -89.514018 \\
Qintana Roo & H58 & 19.584983 & -88.040267 \\
Yucatan & H59 & 21.028224 & -89.507323 \\
\hline
\end{tabular}

Table S6. Latitude and longitude of Biorefinery sites.

\begin{tabular}{|c|c|c|c|}
\hline State & Zone (Node) & Latitude & Longitude \\
\hline Point $(0,0)$ & Origin point & 10.483034 & -110.481251 \\
\hline \multicolumn{4}{|c|}{ Biorefinery Sites } \\
\hline Baja California sur & $\mathrm{R} 1$ & 24.748308 & -111.146436 \\
\hline Baja California sur & $\mathrm{R} 2$ & 27.317561 & -112.764338 \\
\hline Baja California Norte & $\mathrm{R} 3$ & 29.395963 & -114.173949 \\
\hline Baja California Norte & $\mathrm{R} 4$ & 31.402088 & -115.539671 \\
\hline Sonora & R5 & 30.810332 & -112.03848 \\
\hline Sonora & R6 & 30.591404 & -109.549631 \\
\hline Sonora & R7 & 29.21156 & -111.352262 \\
\hline Sonora & $\mathrm{R} 8$ & 28.804551 & -109.61384 \\
\hline Chichuahua & R9 & 30.452459 & -107.364951 \\
\hline Chichuahua & R10 & 30.65974 & -105.71774 \\
\hline Chichuahua & R11 & 28.596558 & -107.194926 \\
\hline Chichuahua & $\mathrm{R} 12$ & 28.630458 & -104.723932 \\
\hline Chichuahua & $\mathrm{R} 13$ & 26.920391 & -106.954995 \\
\hline Chichuahua & $\mathrm{R} 14$ & 27.229991 & -104.834489 \\
\hline Coahulia & $\mathrm{R} 15$ & 28.428787 & -102.595082 \\
\hline Coahulia & R16 & 28.006124 & -101.071694 \\
\hline Coahulia & $\mathrm{R} 17$ & 25.938323 & -102.773153 \\
\hline Coahulia & $\mathrm{R} 18$ & 25.954737 & -101.535475 \\
\hline Nuevo León & R19 & 26.568407 & -100.014914 \\
\hline Nuevo León & $\mathrm{R} 20$ & 24.814753 & -99.9533998 \\
\hline Tamaulipas & $\mathrm{R} 21$ & 25.159308 & -97.9794616 \\
\hline Tamaulipas & $\mathrm{R} 22$ & 23.735745 & -99.0114398 \\
\hline
\end{tabular}




\begin{tabular}{|c|c|c|c|}
\hline Sinaloa & R23 & 25.549363 & -107.982072 \\
\hline Sinaloa & $\mathrm{R} 24$ & 23.497264 & -106.116251 \\
\hline Durango & $\mathrm{R} 25$ & 25.864923 & -105.685938 \\
\hline Durango & $\mathrm{R} 26$ & 25.952076 & -104.171557 \\
\hline Durango & $\mathrm{R} 27$ & 23.868609 & -105.259832 \\
\hline Durango & $\mathrm{R} 28$ & 24.553234 & -104.093552 \\
\hline Zacatecas & $\mathrm{R} 29$ & 23.922236 & -102.948648 \\
\hline Zacatecas & $\mathrm{R} 30$ & 22.738197 & -102.891782 \\
\hline San Luis Potosi & R31 & 22.84331 & -100.976742 \\
\hline San Luis Potosi & $\mathrm{R} 32$ & 22.223318 & -99.5021326 \\
\hline Nayarit & R33 & 21.591319 & -104.799567 \\
\hline Aguascalientes & $\mathrm{R} 34$ & 21.972981 & -102.170742 \\
\hline Guadalajara & $\mathrm{R} 35$ & 20.927764 & -103.636733 \\
\hline Guadalajara & $\mathrm{R} 36$ & 21.126023 & -102.312253 \\
\hline Guadalajara & R37 & 19.855238 & -104.290679 \\
\hline Guadalajara & $\mathrm{R} 38$ & 20.013412 & -102.917443 \\
\hline Guanajuato & R39 & 21.107737 & -101.160208 \\
\hline Queretaro & $\mathrm{R} 40$ & 20.831107 & -99.8495321 \\
\hline Hidalgo & $\mathrm{R} 41$ & 20.401995 & -98.8220093 \\
\hline Veracruz & $\mathrm{R} 42$ & 21.162669 & -97.6338571 \\
\hline Veracruz & R43 & 19.294711 & -96.0864666 \\
\hline Veracruz & R44 & 18.06875 & -94.8196588 \\
\hline Michoacan & $\mathrm{R} 45$ & 19.234286 & -102.272769 \\
\hline Michoacan & $\mathrm{R} 46$ & 19.886838 & -101.008891 \\
\hline Edo y ciudad de Mexico & $\mathrm{R} 47$ & 19.590854 & -99.0864797 \\
\hline Puebla y Tlaxcala & $\mathrm{R} 48$ & 19.406005 & -97.5556991 \\
\hline Guerrero & R49 & 18.448662 & -100.573831 \\
\hline Guerrero & $\mathrm{R} 50$ & 17.513721 & -99.3912407 \\
\hline Oaxaca & R51 & 17.389772 & -96.6146078 \\
\hline Oaxaca & $\mathrm{R} 52$ & 16.525929 & -94.927609 \\
\hline Chiapas & $\mathrm{R} 53$ & 16.730855 & -93.0141404 \\
\hline Chiapas & R54 & 16.384455 & -92.0087271 \\
\hline Tabasco & $\mathrm{R} 55$ & 18.15579 & -92.7782618 \\
\hline Campeche & R56 & 18.760392 & -90.6461038 \\
\hline Campeche & R57 & 18.997304 & -89.4167028 \\
\hline Qintana Roo & $\mathrm{R} 58$ & 19.674915 & -87.9423899 \\
\hline Yucatan & $\mathrm{R} 59$ & 21.118156 & -89.409208 \\
\hline
\end{tabular}


Table S7. Latitude and longitude of Market sites.

\begin{tabular}{cccc}
\hline City & Zone (Node) & Latitude & Longitude \\
\hline Point $(0,0)$ & Origin point & 10.483034 & -110.481251 \\
Camargo & M1 & 27.673598 & -105.151691 \\
Coatzacoalcos & M2 & 17.999915 & -94.557018 \\
Puebla & M3 & 19.262747 & -98.388883 \\
Poza Rica & M4 & 20.500978 & -97.474446 \\
Tamaulipas & M5 & 22.37965 & -97.882346 \\
Salamanca & M6 & 20.5838 & -101.184028 \\
\hline
\end{tabular}

Table S8. Slope $\left(m_{j, n}\right)$ parameters for piecewise function.

\begin{tabular}{ccccc}
\hline $\begin{array}{c}\text { Lignocellulosic } \\
\text { Waste }\end{array}$ & $\mathrm{n} 1$ & $\mathrm{n} 2$ & $\mathrm{n} 2$ & $\mathrm{n} 3$ \\
\hline Corn Stover & 129.53 & 382.49 & 54.589 & 161.21 \\
Wheat Straw & 89.68 & 66.724 & 89.229 & 53.666 \\
$\begin{array}{c}\text { Sorghum } \\
\text { Bagasse }\end{array}$ & 1578.5 & 305.1 & 316.11 & 358.02 \\
$\begin{array}{c}\text { Sugarcane } \\
\text { Bagasse }\end{array}$ & 300.98 & 196.5 & 396.77 & 204.74 \\
\hline
\end{tabular}

Table S9. Intercept $\left(a_{j, n}\right)$ parameters for piecewise function.

\begin{tabular}{ccccc}
\hline $\begin{array}{c}\text { Lignocellulosic } \\
\text { Waste }\end{array}$ & $\mathrm{n} 1$ & $\mathrm{n} 2$ & $\mathrm{n} 2$ & $\mathrm{n} 3$ \\
\hline Corn Stover & 289,234 & $-785,819$ & $2,001 \mathrm{e} 3$ & $-717,641$ \\
Wheat Straw & 247,757 & 345,322 & 154,023 & $1 \mathrm{e} 6$ \\
$\begin{array}{c}\text { Sorghum } \\
\text { Bagasse }\end{array}$ & -296883 & $5 \mathrm{e} 6$ & $5 \mathrm{e} 6$ & $4 \mathrm{e} 6$ \\
$\begin{array}{c}\text { Sugarcane } \\
\text { Bagasse }\end{array}$ & 469475 & 913503 & -788774 & $4 \mathrm{e} 6$ \\
\hline
\end{tabular}


Table S10. Parameters for calculating the utilities cost of biorefineries.

\begin{tabular}{ccc}
$\begin{array}{c}\text { Lignocellulosic } \\
\text { Waste }\end{array}$ & Slope $\left(d_{j}\right)$ & $\begin{array}{c}\text { Intercept }\left(c_{j}\right. \\
\text { ) }\end{array}$ \\
\hline Corn Stover & 84.27 & 643,954 \\
Wheat Straw & 77.171 & 230,773 \\
Sorghum & 138.07 & $4,767,332$ \\
Bagasse & & \\
Sugarcane & 116 & $1.701 \mathrm{e} 6$ \\
Bagasse & & \\
\hline
\end{tabular}

Table S11. Eco Indicator Parameters.

Eco-indicators Lignocellulosic Wastes (points/Ton)

\begin{tabular}{cc}
\hline Corn Stover & 11.34 \\
Wheat Straw & 17.16 \\
Sorghum Bagasse & 5.85 \\
Sugarcane Bagasse & 1.84 \\
\hline Eco-indicators for processing raw materials(points/Ton) \\
\hline Corn Stover & 49.40 \\
Wheat Straw & 44.18 \\
Sorghum Bagasse & 78.68 \\
Sugarcane Bagasse & 50.71 \\
\hline Eco-indicators for transporting ((points*kilometer/Ton) \\
\hline Harvest- Biorefinery & 0.022 \\
Biorefinery-Marker & 0.008 \\
\hline
\end{tabular}

Table S12. Biorefineries and capacities required for the supply chain.

\begin{tabular}{cclc}
\hline State & Zone (Node) & $\begin{array}{c}\text { Raw Material } \\
\text { used. }\end{array}$ & $\begin{array}{c}\text { Biorefinery installed } \\
\text { capacity (Furfural } \\
\text { Ton/yr) }\end{array}$ \\
\hline $\begin{array}{c}\text { Baja California sur } \\
\text { Baja California sur }\end{array}$ & 1 & Corn Stover & 850 \\
Baja California Norte & 3 & Corn Stover & 850 \\
Baja California Norte & 4 & None & 22165 \\
Sonora & 5 & Wheat straw & \\
Sonora & 6 & Corn Stover & 850 \\
Sonora & 7 & Wheat straw & 51000 \\
Sonora & 8 & Wheat straw & 51000 \\
Chichuahua & 9 & Corn Stover & 850 \\
Chichuahua & 10 & Corn Stover & 850 \\
Chichuahua & 11 & Corn Stover & 850 \\
\hline
\end{tabular}




\begin{tabular}{|c|c|c|c|}
\hline Chichuahua & 12 & Corn Stover & 850 \\
\hline Chichuahua & 13 & Corn Stover & 850 \\
\hline Chichuahua & 14 & Corn Stover & 850 \\
\hline Coahulia & 15 & Corn Stover & 850 \\
\hline Coahulia & 16 & Corn Stover & 850 \\
\hline Coahulia & 17 & Corn Stover & 850 \\
\hline Coahulia & 18 & Corn Stover & 850 \\
\hline Nuevo León & 19 & Corn Stover & 850 \\
\hline Nuevo León & 20 & Wheat straw & 3229 \\
\hline Tampico & 21 & Corn Stover & 850 \\
\hline Tampico & 22 & Corn Stover & 850 \\
\hline Sinaloa & 23 & Wheat straw & 7870 \\
\hline Sinaloa & 24 & Wheat straw & 11691 \\
\hline Durango & 25 & Corn Stover & 850 \\
\hline Durango & 26 & Corn Stover & 850 \\
\hline Durango & 27 & Corn Stover & 850 \\
\hline Durango & 28 & Corn Stover & 850 \\
\hline Zacatecas & 29 & Corn Stover & 850 \\
\hline Zacatecas & 30 & Corn Stover & 850 \\
\hline San Luis Potosi & 31 & Corn Stover & 850 \\
\hline San Luis Potosi & 32 & Corn Stover & 2926 \\
\hline Nayarit & 33 & Corn Stover & 850 \\
\hline Aguascalientes & 34 & Corn Stover & 988 \\
\hline Guadalajara & 35 & Corn Stover & 850 \\
\hline Guadalajara & 36 & Wheat straw & 6530 \\
\hline Guadalajara & 37 & Corn Stover & 850 \\
\hline Guadalajara & 38 & Wheat straw & 4353 \\
\hline Guanajuato & 39 & Wheat straw & 29000 \\
\hline Queretaro & 40 & Corn Stover & 2583 \\
\hline Hidalgo & 41 & Corn Stover & 25191 \\
\hline Veracruz & 42 & Corn Stover & 4250 \\
\hline Veracruz & 43 & Corn Stover & 850 \\
\hline Veracruz & 44 & Corn Stover & 25500 \\
\hline Michoacan & 45 & Wheat straw & 5379 \\
\hline Michoacan & 46 & Wheat straw & 12546 \\
\hline Edo y ciudad de Mexico & 47 & Wheat straw & 7156 \\
\hline Puebla y Tlaxcala & 48 & Corn Stover & 25500 \\
\hline Guerrero & 49 & Corn Stover & 25500 \\
\hline Guerrero & 50 & Corn Stover & 25500 \\
\hline Oaxaca & 51 & Corn Stover & 25500 \\
\hline Oaxaca & 52 & Corn Stover & 850 \\
\hline Chiapas & 53 & Corn Stover & 25500 \\
\hline Chiapas & 54 & Corn Stover & 25500 \\
\hline Tabasco & 55 & Corn Stover & 850 \\
\hline Campeche & 56 & Corn Stover & 25500 \\
\hline Campeche & 57 & Corn Stover & 850 \\
\hline
\end{tabular}




\begin{tabular}{cccc}
\hline Quintana Roo & 58 & Corn Stover & 850 \\
Yucatan & 59 & Corn Stover & 850 \\
\hline
\end{tabular}

\section{References}

(1) Ng, R. T. L.; Maravelias, C. T. Design of Biofuel Supply Chains with Variable Regional Depot and Biorefinery Locations. Renew. Energy 2017, 100, 90-102. https://doi.org/10.1016/j.renene.2016.05.009.

(2) Mexican Ministry of Communications and Transport (SCT). Estadística Básica del Autotransporte Federal http://www.sct.gob.mx/fileadmin/DireccionesGrales/DGAF/EST_BASICA/EST_BASIC A_2018/_Estadística_Básica_del_Autotransporte_Federal_2018.pdf.

(3) García-alcaraz, J. L. Tools, Methodologies and Techniques Applied to Sustainable Supply Chains Edited By. 